\title{
Electron Irradiation Cleaning of the SEM and its Samples
}

Andras Vladar ${ }^{1}$, David Hoyle ${ }^{2}$ and Hosoya Kotaro ${ }^{3}$

${ }^{1}$ Natl. Inst. of Standards and Technology, Gaithersburg, Maryland, United States, ${ }^{2}$ Self employed, n/a, Canada, ${ }^{3}$ Hitachi HTA, United States

Deposition of carbonaceous material under electron beam irradiation is an old and persistent problem of scanning electron microscopy. It is an impediment to high-resolution imaging and measurements, especially at the nanometer-scale and at low electron beam landing energies. The emergence of contamination is a complex process of adsorption, dissociation and desorption of carbonaceous molecules. Depending on the kind and amount of precursor molecules, vacuum, sample material and temperature, and the intensity and energy of the irradiating electrons, deposition can overwhelm removal, or the other way. Fortunately, with the introduction and commercial availability of low-energy, plasma-based and other cleaning devices, contamination can be reduced to levels where it is not trivial to detect. [1] Plasma devices, working in low vacuum, which is necessary to start and sustain plasma generation, can effectively remove contamination precursor hydrocarbon molecules to the extent that a clean sample can be imaged and continuously measured for hours without deposition of any perceptible amount of carbonaceous contamination. For checking the effectiveness of the cleaning of the SEM, a reference sample is now available from NIST. [2]

Here we report on the results obtained with a new and effective cleaning device that has recently become available, which is different from plasma cleaning devices. Sparkle EC* (electron cloud) [3] is based on low-energy electron irradiation [4] and it works in high and ultra-high vacuum (UHV), see Figure 1. With thorough removal of contamination precursor molecules, it provides ultra-high cleanliness (UHC) at which no contamination can be detected by SEM imaging, so measurement time can be as long as needed, even hours, the achievable spatial resolution improves significantly, and repeatable electron yield measurements become possible.

Figure 2. illustrates the various levels of cleanliness and vacuum conditions with a NIST Si chip contamination testing reference sample that was irradiated for 10 minutes at it center at twice as high magnification. In case A with ultra-high vacuum (UHV) and clean sample, the sample stays clean after imaging or measurements. In case D, in high vacuum (HV), when the SEM and the sample are dirty, a large number of contamination precursor molecules turned into an ever-growing thick layer of contamination. In case $\mathrm{C}$, in $\mathrm{HV}$, when the SEM is clean, but the sample still has some contamination precursor molecules, the typical contamination frame appears, but in the center where desorption overwhelms adsorption, so the sample stays clean. In case B, in HV, in UHC condition, when the SEM and the samples are both clean, no contamination appears. Note that the center of the Si sample gets slightly brighter due formation of Si oxide [5] in the presence of water on the surface.

Low-energy electron irradiation-based cleaning of the SEM sample chamber and/or its sample is an effective technique with advantages over other cleaning techniques used for the elimination of precursor molecules of electron-beam-induced contamination. Its fast application and treatment times, small size, and excellent compatibility with SEM practice make it a candidate to be designed for and installed in SEMs to work whenever it is necessary or on a regular basis to ensure that the SEM always works in the state of ultra-high cleanliness, and it can provide excellent results. 


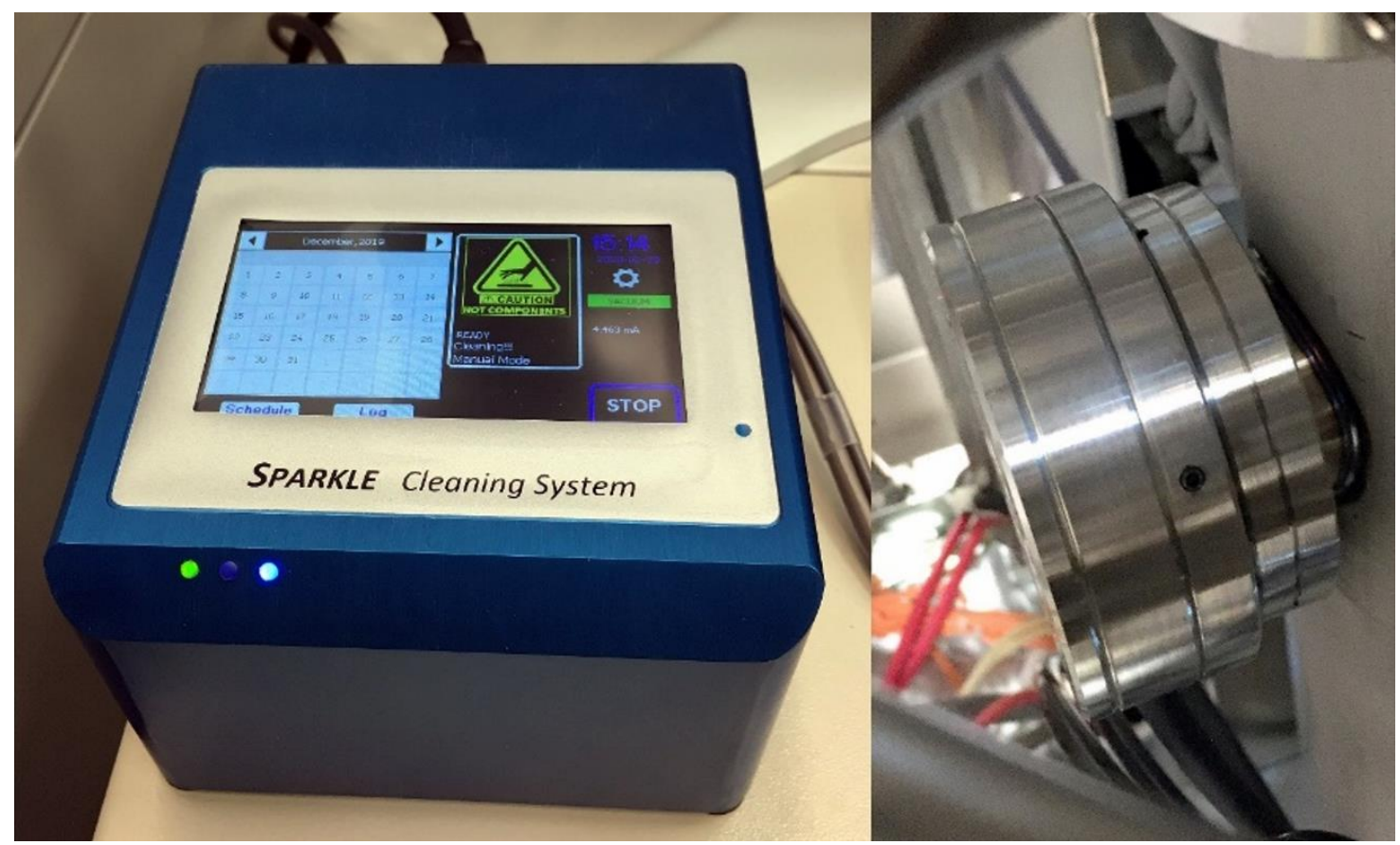

Figure 1. Figure 1. The programmable control unit of the electron irradiation-based cleaning system (left) and the $80 \mathrm{~mm}$ diameter cleaning head (right), the flood-type electron source.

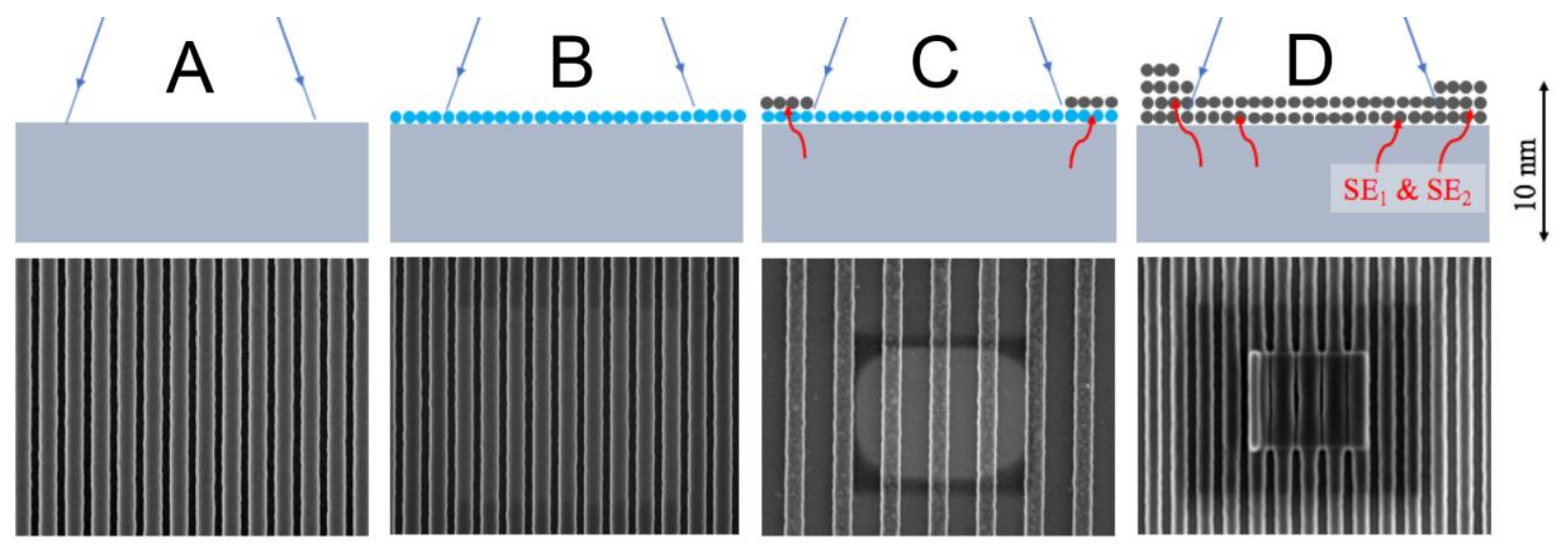

Figure 2. Figure 2. Sample cleanliness after 10 minutes of irradiation at the center of a Si chip sample. The various states illustrated by A UHV, B HV, UHC, C HV, dirty sample, D HV, dirty sample, dirty SEM cases. A, B, C: 1.27 micrometer horizontal field width (HFW), D: HFW 2.45 micrometer, $1 \mathrm{keV}$ landing energy, $43 \mathrm{pA}$ beam current, 1-minute imaging times, 10-minute irradiation times at the center.

\section{References}

1. Active monitoring and control of electron-beam-induced contamination, 2001, A. E. Vladár, et al. https://doi.org/10.1117/12.436724

2. The Scanning Electron Microscope Contamination Assessment Reference Sample (SEMCARS) is available for free through a cooperation with NIST, contact the author at andras@ nist.gov

3. Sparkle EC of Hitachi High-Technologies Canada, Inc. https://www.hitachi-hightech.com/ca/

4. Contamination Mitigation Strategy for Ultra-Low Energy Electron Microscopy and Spectroscopy, 2019, E. M. Mikmeková et al. https://doi.org/10.1017/S1431927619003234

5. Electron irradiation induced amorphous $\mathrm{SiO} 2$ formation at metal oxide/Si interface at room temperature; electron beam writing on interfaces, 2018, S. Gurbán, et al. https://doi.org/10.1038/s41598-018-20537-4 